\title{
Direct Measurement of Quantum Confinement Effects at Metal to Quantum-Well Nanocontacts
}

\author{
C. Tivarus and J. P. Pelz \\ Department of Physics, The Ohio State University, Columbus, Ohio 43210-1106, USA \\ M. K. Hudait and S. A. Ringel \\ Department of Electrical and Computer Engineering, The Ohio State University, Columbus, Ohio 43210-1106, USA
}

(Received 31 August 2004; published 23 May 2005)

\begin{abstract}
Model metal-semiconductor nanostructure Schottky nanocontacts were made on cleaved heterostructures containing GaAs quantum wells (QWs) of varying width and were locally probed by ballistic electron emission microscopy. The local Schottky barrier was found to increase by $\sim 0.140 \mathrm{eV}$ as the QW width was systematically decreased from 15 to $1 \mathrm{~nm}$, due mostly to a large $(\sim 0.200 \mathrm{eV})$ quantumconfinement increase to the QW conduction band. The measured barrier increase over the full 1 to $15 \mathrm{~nm}$ QW range was quantitatively explained when local "interface pinning" and image force lowering effects are also considered.
\end{abstract}

DOI: 10.1103/PhysRevLett.94.206803

Much recent activity has focused on nanoscale semiconducting devices [1-5]. Critical to their behavior are the contacts that transport carriers in and out of the device. For example, some argue that the device behavior of carbon nanotube field-effect transistors is dominated by Schottky barriers (SBs) that form at the metal contacts to the nanotube [4,5]. In nm-dimension Schottky contacts, small-size effects - such as quantum confinement and geometryinduced electric fields - may greatly alter carrier transport through the contacts. There is a critical need to predict how these effects scale with contact size and geometry. Several previous studies have measured current-voltage curves through nm-sized metal contacts on homogenous semiconductor (SC) substrates, to infer effects from nonlocal "environmental pinning" and geometry-induced electric fields $[6,7]$. However, those contacts were not to semiconducting nanostructures, so effects such as quantum confinement were negligible. Furthermore, the small-size effects on the Schottky barrier were inferred from current-voltage measurements, and not directly measured with a spectroscopic technique.

Here we report nm-resolution measurements of carrier transport through a model metal-SC nanostructure system, in which the nanostructure dimension can be systematically varied down to $1 \mathrm{~nm}$. This model system is made on the cleaved edge of a GaAs wafer containing a series of $\mathrm{AlGaAs} / \mathrm{GaAs} / \mathrm{AlGaAs}$ quantum wells (QWs) of different thickness. The measured quantity is the energy offset between the metal Fermi energy and the SC conduction band minimum: the Schottky barrier height $(\mathrm{SBH})$. The measurement tool is ballistic electron emission microscopy (BEEM) $[8,9]$, which directly measures the local SBH with nanoscale spatial resolution and $<20 \mathrm{meV}$ energy resolution. The SBH shows a strong $140 \mathrm{meV}$ systematic increase as the QW width $d$ is reduced to $1 \mathrm{~nm}$. Our measurements agree very well with the predicted increase from simple one-dimensional quantum confinement, adjusted by a smaller calculated decrease $(\sim 70 \mathrm{meV}$ for a
PACS numbers: 73.21.Fg, 73.30.+y, 73.63.Hs, 73.63.Rt

$1 \mathrm{~nm}$ QW) due to environmental pinning effects [6,7] and increased image force lowering [10].

The samples consisted of a sequence of GaAs QWs $\left(\sim 5 \times 10^{16} / \mathrm{cm}^{3} n\right.$-type $)$ with width varying between 1 and $15 \mathrm{~nm}$, separated by $200 \mathrm{~nm}$ thick $\mathrm{Al}_{0.3} \mathrm{Ga}_{0.7}$ As barrier layers ( $\sim 1 \times 10^{17} / \mathrm{cm}^{3} n$-type), grown by molecular beam epitaxy on a $200 \mathrm{~nm}$ thick GaAs buffer layer $(\sim 2-3 \times$ $10^{15} / \mathrm{cm}^{3} n$-type $)$ on a $\mathrm{GaAs}(001)$ substrate $(\sim 3 \times$ $10^{17} / \mathrm{cm}^{3} n$-type). A $1.5 \mu \mathrm{m} \mathrm{GaAs}$ capping layer with the same doping as the QWs was used as a wide GaAs reference layer. The wafers were cleaved ex situ along a [110] direction, and $\sim 300 \mu \mathrm{m}$ diameter Au Schottky contacts (of thickness 4 or $7 \mathrm{~nm}$ ) were deposited through a shadow mask or using lift-off photolithography, by electron-beam evaporation at a typical pressure of $2 \times$ $10^{-7}$ torr. For the lift-off process, the cleaved surface was first treated in a 1:1 $\mathrm{NH}_{4} \mathrm{OH}: \mathrm{H}_{2} \mathrm{O}$ etching solution for $1 \mathrm{~min}$ followed by a rinse in deionized $\mathrm{H}_{2} \mathrm{O}$. This process caused the QWs to be raised by $\sim 0.5 \mathrm{~nm}$ relative to the surrounding $\mathrm{Al}_{0.3} \mathrm{Ga}_{0.7}$ As surface, due to the faster oxidation rate of $\mathrm{Al}_{0.3} \mathrm{Ga}_{0.7} \mathrm{As}$ and the subsequent etch of this oxide [11]. The raised QWs in this case altered the local grain growth during $\mathrm{Au}$ deposition [see Fig. 1(c)]. With the shadow-mask preparation, no such QW-related topographic features were observed after the metal deposition [Fig. 1(a)]. Samples were then transferred into a BEEM-modified ultrahigh vacuum Omicron VT scanning tunneling microscopy (STM) system for room temperature measurements. We note that Talin et al. [12] reported that the thin interfacial oxide layer that forms during air exposure (prior to metal deposition) does not affect BEEM measurements and that samples with native oxide layers are more uniform than the ones prepared in situ.

In BEEM (an extension of STM), an STM tip held at a voltage $\left(-V_{T}\right)$ relative to the metal overlayer film injects hot electrons (with total current $I_{T}$ ) into a metal, with an energy distribution with maximum energy $\cong e V_{T}$ above the metal Fermi energy. Provided the metal layer is suffi- 


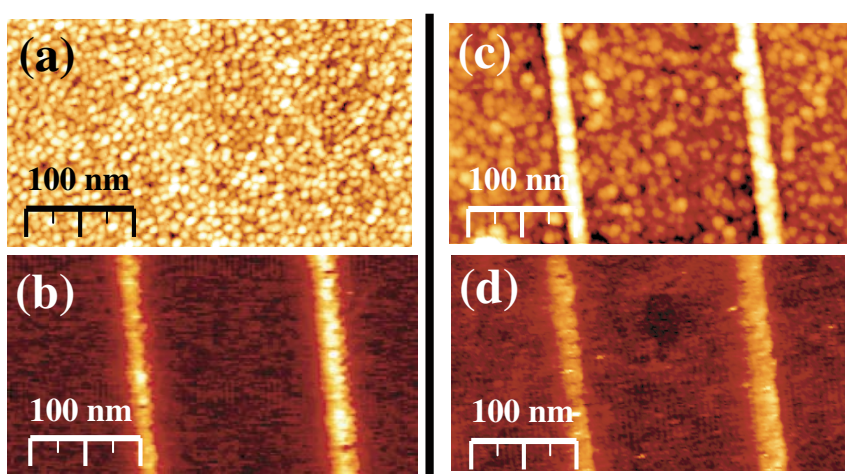

FIG. 1 (color). (a) STM image of the top $7 \mathrm{~nm}$ thick Au film over a $7 \mathrm{~nm}$ (left) and $9 \mathrm{~nm}$ (right) QW for a shadow-mask sample and (b) a simultaneous BEEM image of the same area. The QWs are visible only in (b). (c) A STM and (d) a simultaneous BEEM image of a $7 \mathrm{~nm}$ Au film made with photolithography. The 7 and $9 \mathrm{~nm}$ QWs are visible in (c) because the surface preparation causes the QWs to be raised (see the text). Color scale: $4.6 \mathrm{~nm}$ for (a), $2.5 \mathrm{~nm}$ for (c), and 0-4 pA for (b) and (d). All data taken with $V_{T}=1.15 \mathrm{~V}$ and $I_{T}=15 \mathrm{nA}$.

ciently thin $(<\sim 25 \mathrm{~nm}$ for $\mathrm{Au})$ a small fraction of injected electrons will transverse the metal layer ballistically, and if they have sufficient energy can enter the SC conduction band and be "collected" as the BEEM current $I_{C}$. The local SBH can be determined from the threshold behavior of "BEEM spectra," which measure the dependence of $I_{C}$ as a function of $V_{T}$ [8].

Figures 1 and 2 demonstrate that BEEM can locate the subsurface QWs and then quantify the size-dependent SBH over the QWs. Figure 1(a) shows a $400 \times 200 \mathrm{~nm}$ STM topographic image of a polycrystalline $7 \mathrm{~nm}$ thick Au film deposited using the shadow mask, over a sample region with a $7 \mathrm{~nm}$ (left) and a $9 \mathrm{~nm}$ (right) QW. Figure 1(b) shows the simultaneously acquired "BEEM image" (i.e., a plot of $I_{C}$ vs tip position) of the same area, with $V_{T}=1.15 \mathrm{~V}$ and tunnel current $I_{T}=15 \mathrm{nA}$. The subsurface $\mathrm{QWs}$ are clearly visible in Fig. 1(b) as regions of enhanced BEEM current, due to a lower local SBH over the QWs as compared with the surrounding $\mathrm{Au} / \mathrm{Al}_{0.3} \mathrm{Ga}_{0.7} \mathrm{As}$ interface. Figures 1(c) and 1(d) show similar STM and BEEM images for a sample prepared by photolithography. In this case the QWs are also visible in the STM image due to the chemical treatment discussed above. However, the two BEEM images look very similar, suggesting (and later confirmed) that both treatments result in essentially the same metal-QW contact properties. We also note that the apparent width of each QW in the BEEM images is larger than the actual QW width, which is mainly due to lateral broadening of the hot electron beam in the Au film (due to scattering [13]) before they cross into the SC. A detailed study of this beam broadening will be presented elsewhere. Note also that a small amount of BEEM current is observed over the $\mathrm{Al}_{0.3} \mathrm{Ga}_{0.7}$ As layers since $V_{T}$ is slightly above the 1.085. eV Au/ $/ \mathrm{Al}_{0.3} \mathrm{Ga}_{0.7}$ As SBH [see Fig. 2(d)].
Figure 2(a) compares averaged BEEM spectra measured with the tip located over four QWs of different widths, with the spectra measured over the wide GaAs capping layer (far left curve) and over a wide $\mathrm{Al}_{0.3} \mathrm{Ga}_{0.7}$ As barrier layer (far right curve). These spectra were measured on the photolithography-defined sample. Essentially the same results were found on shadow-mask samples, except for a very small $10-20 \mathrm{meV}$ systematic increase in the measured SBH for all sample regions. Least-squares fits to the Bell-Kaiser model [8] (solid lines) provided a good fit to the data and were used to determine the local SBHs. We note that the Bell-Kaiser model includes Fermi broadening of electrons inside the STM tip, and hence can fit the small amount of "subthreshold" $I_{C}$ that can be observed down to several $k_{B} T$ below the actual SBH. The BEEM spectrum for the reference GaAs region had two fitted thresholds at $\sim 0.900 \mathrm{eV}$ and $\sim 1.200 \mathrm{eV}$ (not shown), corresponding to $\Gamma$ and $L$ points of the GaAs conduction band, in good accordance with previous results [14]. The spectrum for the $\mathrm{Al}_{0.3} \mathrm{Ga}_{0.7} \mathrm{As}$ barrier layers also had two thresholds at $\sim 1.085$ and $\sim 1.210 \mathrm{eV}$, respectively, which is $\sim 60 \mathrm{meV}$ lower than other reports for a similar Al fraction $[15,16]$. We point out that different surface treatments can result in different SBH values [15], and previous studies used a
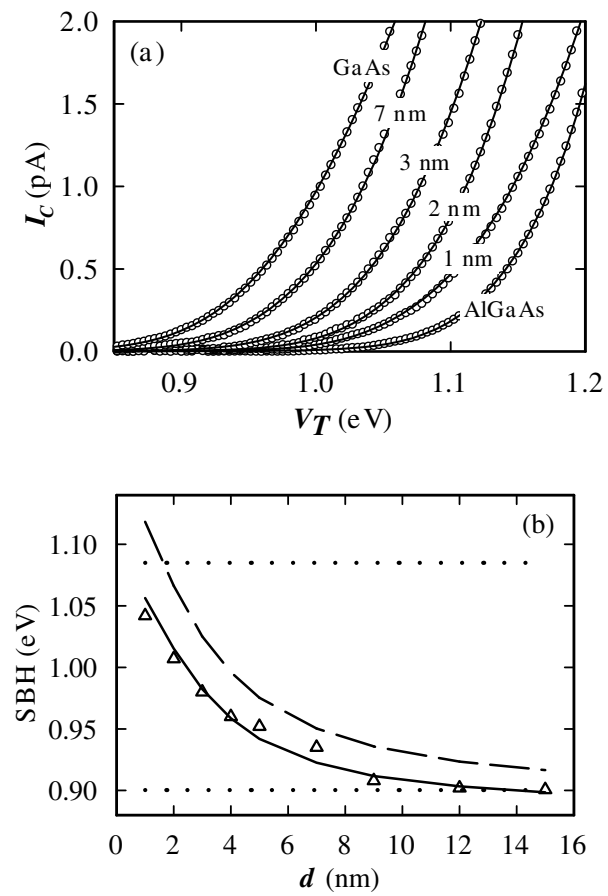

FIG. 2. (a) Scaled averaged BEEM spectra (empty circles) and Bell-Kaiser fits to the data (solid lines). From left to right: $\mathrm{Au} /$ reference $\mathrm{GaAs}$ (scaled relative to $\mathrm{Au} / \mathrm{Al}_{0.3} \mathrm{Ga}_{0.7} \mathrm{As}$ by a factor $\mathrm{F}=0.4), 7 \mathrm{~nm} \mathrm{QW}(\mathrm{F}=2), 3 \mathrm{~nm} \mathrm{QW}(\mathrm{F}=1.8), 2 \mathrm{~nm} \mathrm{QW}$ $(\mathrm{F}=1.7), 1 \mathrm{~nm} \mathrm{QW}(\mathrm{SF}=1.1), \mathrm{Au} / \mathrm{Al}_{0.3} \mathrm{Ga}_{0.7} \mathrm{As}$. (b) $\mathrm{SBH}$ as a function of QW width: experimental data (empty triangles), simple 1D model (dashed line), and full model (solid line). The dotted lines show $\mathrm{SBH}$ for uniform $\mathrm{Au} / \mathrm{Al}_{0.3} \mathrm{Ga}_{0.7} \mathrm{As}$ (top) and $\mathrm{Au} / \mathrm{GaAs}$ (bottom). 
(100) wafer orientation, while our measurements were made on a cleaved (110) plane. We note here that when the tip is directly over a $\mathrm{QW}$ and $V_{T}>1.085 \mathrm{eV}$, then lateral hot electron spreading in the Au film will cause some of the hot electrons to cross over the neighboring $\mathrm{Au} / \mathrm{Al}_{0.3} \mathrm{Ga}_{0.7} \mathrm{As}$ interface and contribute to the measured $I_{C}$. As a result, the BEEM spectra over the QWs will be the superposition of two BEEM currents [9], one due to the QW and the other due to the neighboring $\mathrm{Al}_{0.3} \mathrm{Ga}_{0.7} \mathrm{As}$ region.

To extract the SBH from the spectra measured over the QWs, we used the Bell-Kaiser model with two thresholds, with the second threshold held fixed at the already measured $\mathrm{Al}_{0.3} \mathrm{Ga}_{0.7} \mathrm{As} \mathrm{SBH}$. In this case we restrict the data range to values which are in a voltage range lower than $1.150 \mathrm{eV}$, in order to avoid overlap with the second thresholds of $\mathrm{Au} / \mathrm{GaAs}$ and $\mathrm{Au} / \mathrm{Al}_{0.3} \mathrm{Ga}_{0.7} \mathrm{As}$, which are both close to $\sim 1.200 \mathrm{eV}$. As a check, we also performed a single threshold fit of the part of the data below the $\mathrm{Al}_{0.3} \mathrm{Ga}_{0.7} \mathrm{As} \mathrm{SBH}$ of $\sim 1.085 \mathrm{eV}$, for which the amplitude of the $\mathrm{Al}_{0.3} \mathrm{Ga}_{0.7}$ As signal is zero. The results of the two fitting procedures were within $5 \mathrm{meV}$ of each other. Because of the hot-electron spreading in the Au film fewer electrons can enter the narrower QWs, resulting in a reduced signal-to-noise ratio for these spectra. To check whether the signal-to-noise ratio affects our fits, we made measurements of samples with a thinner $4 \mathrm{~nm}$ thick $\mathrm{Au}$ film and used up to $I_{T}=25 \mathrm{nA}$, both of which significantly increased the signal-to-noise ratio. In all cases, the extracted threshold voltages were independent of $I_{T}$ and Au film thickness to within $10 \mathrm{meV}$.

The data points (triangles) in Fig. 2(b) show the measured $\mathrm{SBH}$ as a function of the QW width $d$, determined by fitting the BEEM spectra shown in Fig. 2(a). We see that the SBH increases systematically with decreasing $Q W$ width up to $\sim 140 \mathrm{meV}$ for $d \cong 1 \mathrm{~nm}$. In order for the injected hot electrons to pass from the metal into the semiconductor and be collected as $I_{C}$ they must enter available propagating states within the SC conduction band. For the QWs these available states are the GaAs conduction band states, modified by quantum confinement (from the AlGaAs barriers), interface states near the $\mathrm{Au} / \mathrm{QW}$ interface (see below), and the moderately strong $\left(\sim 2 \times 10^{5} \mathrm{~V} / \mathrm{cm}\right)$ "depletion field" that exists near the metal interface of a SB [10] (see below). As a first estimate of these effects, we have calculated the quantumconfinement energy expected for a simple one-dimensional (1D) particle-in-a-box model, which neglects the interface states and the depletion field. The parameters used in this 1D model are electron effective mass $m^{*} \cong 0.067 m_{0}$ for the $\mathrm{GaAs}$ and $m^{*} \cong 0.092 m_{0}$ for the surrounding $\mathrm{Al}_{0.3} \mathrm{Ga}_{0.7} \mathrm{As}$ barrier layer [17], with $m_{0}$ the free electron mass, and a conduction band offset $\Delta E_{C B} \cong 0.250 \mathrm{eV}$ between $\mathrm{GaAs}$ and $\mathrm{Al}_{0.3} \mathrm{Ga}_{0.7}$ As [18]. The dashed line in Fig. 2(b) shows the sum of this calculated 1D quantum- confinement energy and the measured SBH for the uniform $\mathrm{Au} / \mathrm{GaAs}$ interface. We see that even this simple model tracks the measured data fairly well, although it systematically overestimates the actual increase in SBH. This demonstrates that quantum confinement has a strong local effect on the SBH of these contacts, which can be directly observed by BEEM.

To account for the effects of interface states, we have calculated the potential distribution near the $\mathrm{Au} / \mathrm{SC}$ interface close to a QW using the FLEXPDE finite-element solver [19]. It is well-known that both $\mathrm{Au} / \mathrm{GaAs}$ and $\mathrm{Au} / \mathrm{Al}_{0.3} \mathrm{Ga}_{0.7} \mathrm{As}$ interfaces are partially "pinned," due to the presence of interface states near the metal-SC interface whose charge depends on the local Fermi energy $[10,13,17]$. Figure 3 is a sketch of the model geometry. The charge in the interface states is positive for both $\mathrm{Au} / \mathrm{GaAs}$ and $\mathrm{Au} / \mathrm{Al}_{0.3} \mathrm{Ga}_{0.7} \mathrm{As}$ but is larger for the more strongly pinned $\mathrm{Au} / \mathrm{Al}_{0.3} \mathrm{Ga}_{0.7} \mathrm{As}$ interface [13,17]. This charge (shown as "+" symbols in Fig. 3) and its image charge in the metal film (shown as "-" symbols) produce a potential drop across a thin "interfacial layer" at the metal-SC interface, thus altering the effective SBH [10]. However, for narrow QWs, the larger interface charge on the surrounding $\mathrm{Au} / \mathrm{Al}_{0.3} \mathrm{Ga}_{0.7} \mathrm{As}$ interface increases the potential drop over the QWs and hence slightly lowers the measured SBH. This effect is similar to the environmental pinning effects that have been considered for other metalSC contact geometries [6,7].

To quantify this, we assumed a set of interface parameters based on previous studies [13,17], which give the measured $\mathrm{SBH}$ and interface pinning strength for uniform $\mathrm{Au} / \mathrm{GaAs}$ and $\mathrm{Au} / \mathrm{Al}_{0.3} \mathrm{Ga}_{0.7} \mathrm{As}$ interfaces. For GaAs $\left(\mathrm{Al}_{0.3} \mathrm{Ga}_{0.7} \mathrm{As}\right)$ the assumed parameters are $[10,13,17,20]$ charge neutrality level $\mathrm{CNL}=$ $0.53 \mathrm{eV}(0.70 \mathrm{eV})$ above the valence band maximum, interface states density $D_{s}=1.25 \times 10^{18} / \mathrm{m}^{2} \quad(2.65 \times$ $\left.10^{18} / \mathrm{m}^{2}\right)$, electron affinity $\chi=4.07 \mathrm{eV}(3.74 \mathrm{eV})$, dielectric coefficient $\quad \varepsilon_{r}=13.1(12.2), \quad$ band gap $=$ $1.423 \mathrm{eV}(1.845 \mathrm{eV}), \mathrm{Au}$ work function $=5.1 \mathrm{eV}$, and a $0.4 \mathrm{~nm}$ thick interfacial layer with the free-space dielectric

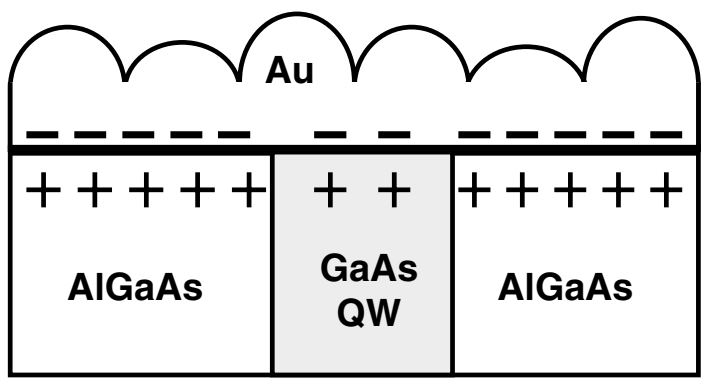

FIG. 3. A schematic diagram of the geometry used for the theoretical simulation. The + symbols represent the positive interface state charge, which is larger on the metal- $\mathrm{Al}_{0.3} \mathrm{Ga}_{0.7} \mathrm{As}$ interface than on the metal-QW interface. The - symbols represent the image charge in the metal film. 
constant $\varepsilon_{0}$ [10]. With these parameters, the calculated reduction in the QW conduction band minimum (relative to uniform $\mathrm{Au} / \mathrm{GaAs}$ ) is small, ranging from $\sim 5 \mathrm{meV}$ for $d=15 \mathrm{~nm}$ to $\sim 30 \mathrm{meV}$ for $d=1 \mathrm{~nm}$. This would tend to slightly lower the predicted SBH curve in Fig. 2(b).

The depletion electric field $E_{d}$ at the metal-SC interface can also affect the measured SBH through the well-known "image force lowering" (IFL) effect [10], which results from the depth-dependent reduction in an electron's potential due to its image charge in the metal. This results in a lowering of the SBH by an amount equal to $\Delta \mathrm{SBH}_{\mathrm{IFL}}=$ $-e \sqrt{e E_{d} / 4 \pi \varepsilon_{r} \varepsilon_{0}}$, where $e$ is the elementary charge [10]. Our modeling indicates that the depletion electric field is stronger in the QWs as compared with a uniform $\mathrm{Au} / \mathrm{GaAs}$ SB contact and is strongest for the narrowest QWs. This would slightly increase the IFL for the $\mathrm{Au} / \mathrm{QW}$ contacts. There are two reasons for this larger electric field. First, the uniform $\mathrm{Au} / \mathrm{Al}_{0.3} \mathrm{Ga}_{0.7} \mathrm{As} \mathrm{SB}$ contact has a larger depletion field than the uniform $\mathrm{Au} / \mathrm{GaAs}$ contact, due to its larger barrier height $(1.085$ vs $0.900 \mathrm{eV})$ and larger doping $(1 \times$ $10^{17}$ vs $\left.5 \times 10^{16} / \mathrm{cm}^{3}\right)$. A GaAs QW embedded in the $\mathrm{Au} / \mathrm{Al}_{0.3} \mathrm{Ga}_{0.7} \mathrm{As}$ will "feel" this stronger depletion field, provided the QW width is smaller than the $\mathrm{Au} / \mathrm{Al}_{0.3} \mathrm{Ga}_{0.7} \mathrm{As}$ depletion width. In addition, the environmental pinning discussed above will further increase the local electric field very close to the $\mathrm{Au} / \mathrm{QW}$ interface, with a stronger enhancement for the narrower QWs. Our modeling indicates $\Delta \mathrm{SBH}_{\mathrm{IFL}} \cong-35 \mathrm{meV}$ for a uniform $\mathrm{Au} / \mathrm{GaAs}$ contact, $-47 \mathrm{meV}$ for a $15 \mathrm{~nm} \mathrm{Au} / \mathrm{QW}$ contact, and $-69 \mathrm{meV}$ for a $1 \mathrm{~nm} \mathrm{Au} / \mathrm{QW}$ contact. The change in IFL relative to a uniform $\mathrm{Au} / \mathrm{GaAs}$ interface is smallonly $\sim 12-35 \mathrm{meV}$ - but should produce an observable reduction in the SB measured by BEEM.

The solid line in Fig. 2(b) includes the sum of the SB increase due to $1 \mathrm{D}$ quantum confinement, and the smaller decreases due to environmental pinning and IFL that were discussed above. The overall agreement between this refined model and the data is very good. One puzzle is the apparent small $\sim 10 \mathrm{meV}$ "bump" in the measured SB for the 5 and $7 \mathrm{~nm}$ QWs. This particular behavior was reproduced on separate diodes, with different Au film thickness, so we do not think that is simply due to spurious measurements. However, since all the QW samples we have studied came from the same wafer, even very minor variations in the average QW thickness - as low as $\sim 0.3 \mathrm{~nm}$ - could produce these observations. Additional work is necessary to specify the actual source.

In summary, we have directly measured the dependence of the SBH on SC nanostructure size down to $1 \mathrm{~nm}$, using cross-sectional BEEM on cleaved QW structures. The local SBH increases systematically with decreasing QW width up to $\sim 140 \mathrm{meV}$ for $d=1 \mathrm{~nm}$, which is quantitatively explained by a large $(\sim 200 \mathrm{meV})$ increase due to quantum confinement, modified by smaller decreases due to environmental pinning and IFL. Finally, we note that it should be possible to make similar local measurements of the SBH on other metal-SC nanostructure contacts, provided the top metal film is thin and accessible to an STM probe tip.

The authors thank R. Goldman and R. Feenstra for helpful discussions. This work was supported by the Office of Naval Research under Grant No. N00014-93-10607 and by the National Science Foundation under Grants No. DMR-0076362 and No. DMR-0313468.

[1] Y. Cui and C. M. Lieber, Science 291, 851 (2001).

[2] F. Leonard and J. Tersoff, Phys. Rev. Lett. 84, 4693 (2000).

[3] Y. Zhang and T. Ichihashi, Science 285, 1719 (1999).

[4] S. Heinze, J. Tersoff, R. Martel, V. Derycke, J. Appenzeller, and Ph. Avouris, Phys. Rev. Lett. 89, 106801 (2002).

[5] J. Appenzeller, J. Knoch, V. Derycke, R. Martel, S. Wind, and Ph. Avouris, Phys. Rev. Lett. 89, 126801 (2002).

[6] H. Hasegawa, T. Sato, and C. Kaneshiro, J. Vac. Sci. Technol. B 17, 1856 (1999).

[7] G. D. J. Smit, S. Rogge, and T. M. Klapwijk, Appl. Phys. Lett. 80, 2568 (2002); Microelectron. Eng. 64, 429 (2002).

[8] W. J. Kaiser and L.D. Bell, Phys. Rev. Lett. 60, 1406 (1988); 61, 2368 (1988).

[9] We have recently demonstrated that BEEM can also quantify the local Schottky barrier height over selfforming quantum wells in SiC: Y. Ding, K. B. Park, J. P. Pelz, K. C. Palle, M. K. Mikhov, B. J. Skromme, H. Meidia, and S. Mahajan, Phys. Rev. B 69, 041305 (2004).

[10] S. M. Sze, Physics of Semiconductor Devices (John Wiley \& Sons, Inc., New York, 1969).

[11] F. Reinhardt, B. Dwir, and E. Kapon, Appl. Phys. Lett. 68, 3168 (1996).

[12] A. A. Talin, D. A. Ohlberg, R. S. Williams, P. Sullivan, I. Koutselas, B. William, and K. L. Kavanagh, Appl. Phys. Lett. 62, 2965 (1993).

[13] L. D. Bell, Phys. Rev. Lett. 77, 3893 (1996).

[14] D. L. Smith, E. Y. Lee, and V. Narayanamurti, Phys. Rev. Lett. 80, 2433 (1998).

[15] G. M. Vanalme, L. Goubert, R. L. Van Meirhaeghe, F. Cardon, and P. Van Dale, Semicond. Sci. Technol. 14, 871 (1999).

[16] X.-C. Cheng, D. A. Collins, and T. C. McGill, J. Vac. Sci. Technol. A 15, 2063 (1997).

[17] P. Roblin and H. Rohdin, High-Speed Heterostructure Devices (Cambridge University Press, Cambridge, England, 2002).

[18] J. J. O'Shea, E. G. Brazel, M. E. Rubin, S. Bhargava, M. A. Chin, and V. Narayanamurti, Phys. Rev. B 56, 2026 (1997).

[19] www.pdesolutions.com.

[20] R.E. Allen, T. J. Humphreys, and J. D. Dow, Solid State Commun. 49, 1 (1983). 